\title{
Psychophysical scales of apparent heaviness and the size-weight illusion ${ }^{1}$
}

\author{
JOSEPH C. STEVENS ${ }^{2}$ AND LEE L. RUBIN ${ }^{3}$ \\ JOHN B. PIERCE FOUNDA TION LABORATORY AND YALE UNIVERSITY
}

\begin{abstract}
The apparent heaviness of a set of 40 cylindrical objects was scaled by the method of magnitude estimation. The objects varied in weight, volume, and density. There were three main conclusions: (1) For any constant volume, heaviness grows as a power function of weight; the larger the volume, the larger the exponent of the power function. The family of such power functions converge at a common point in the vicinity of the heaviest weight that can be lifted. (2) For any constant density (i.e., weight proportional to volume). heaviness does not grow as a power function of weight. (3) For any constant weight, heaviness decreases approximately as a logarithmic function of volume; the constants of the log function depend systematically on the weight of the object. The outcome furnishes a broad quantitative picture of apparent heaviness and of the size-weight illusion (Charpentier's illusion).
\end{abstract}

This paper describes how apparent heaviness depends on the weight, volume, and density of lifted objects. Two lines of research converge here: (1) psychophysical scaling of apparent heaviness as a function of weight, e.g., the veg scale as developed by S. S. Stevens and Galanter (1957); and (2) measurement of the size-weight illusion, often called Charpentier's illusion, the observation that the larger the volume over which a given weight is distributed, the lighter the object feels. Some investigations of apparent heaviness have been questioned (e.g., by Warren \& Warren, 1956) on the grounds that the size-weight illusion may have influenced thelı.

The exact nature and extent of the influence cannot be easily inferred, however, from the bulky literature on the illusion. It has long been known that the illusion ".. . is greater with small weights than with large weights" (Wolfe, 1898, p. 35), but what seems needed is a quantification of the illusion over large ranges of weight and volume. Though this might appear to call for a massive and tedious matching program, numerical scaling methods, e.g., magnitude estimation, can be used to facilitate the measurements. The usefulness of numerical matching methods in mapping families of psychophysical functions involving several parameters has already been demonstrated. Examples include the measurement of apparent force of isometric contraction as a function of both the duration and level of tension (J. C. Stevens \& Cain, in press); the measurement of apparent warmth as a function of the duration, level, and areal extent of infrared irradiation of the skin ( $\mathrm{J}$. C. Stevens \& Marks, in press); and the measurement of apparent loudness and brightness as functions of the intensity and duration of the stimulus (J. C. Stevens \& Hall, 1966). Those and other successes encouraged us to try the method of magnitude estimation in a parametric study of heaviness in which the stimuli varied along the dimensions of weight, volume, and density. The purpose was to answer three questions: (1) For any given (constant) volume, how does apparent heaviness grow with physical weight? Does the growth rate depend on volume? (2) For any given (constant) density, how does apparent heaviness depend on weight? (3) For any given (constant) weight, how does apparent heaviness decrease with increased volume? Does the rate of decrease depend on weight?

\section{APPARATUS}

The stimulus objects all had approximately the same cylindrical shape; the ratio of circumference to height equaled about 1.8 . They were constructed from polyethylene bottles (Macalaster-Bicknell Co. Series 3512) that ranged in nominal volume from $1 \mathrm{oz}$ to $0.5 \mathrm{gal}$ ( 30 to $2,000 \mathrm{ml}$ ). A round wooden knob ( $3 \mathrm{~cm}$ in diam) of the type used on a chest of drawers, was attached to the screw cap of each bottle; the weight was lifted by this knob. The outsides of the bottles were painted gray to make them completely opaque. To adjust the weight, small pieces of lead and cotton batting were distributed uniformly inside the bottle; grossly nonuniform distribution can influence apparent heaviness (Koseleff, 1957-58). The volume of the entire object, including the knob, was measured by immersion in water.

Altogether there were 40 stimulus objects having a range from 58 to $2,325 \mathrm{ml}$ in volume and a range from 25 to $6,400 \mathrm{~g}$ in weight. Table 1 specifies the weights and volumes.

\section{PROCEDURE}

Each of 11 men and three women served in two test sessions, separated by at least a day. The two sessions were identical, except for the order in which the stimulus objects were given to the $S$. In every session the order was irregular with respect to both volume and weight.

The $S$ sat at a table in a chair of adjustable height. The height was adjusted to give him good leverage on the weights. The $S$ could see only the particular weight placed before him on the table.

The following instructions were read at each session.

"You will be asked to estimate the apparent heaviness of various weights, which you will lift with your preferred hand.

"How to lift the weights: Grasp each weight by the wooden handle at the top and lift for a second or two at a time. Please do not hold for a long time, or shake, or tilt the weight. If you wish, you may lift the weight more than once before making your estimate.

"How to make the estimations: Assign a convenient number to the first weight to stand for its apparent heaviness. To the subsequent weights (some of which will be heavier, some lighter than the first) assign other numbers in proportion to apparent

Table 1

The Matrix of Geometric Means of the Magnitude Estimations of Heaviness of Stimulus Objects of Various Weights and Volumes. The rows show constant weight, the columns show constant volume, and the diagonals show roughly constant density. Each cell is the geometric mean of 28 estimations ( 2 from each of 14 Ss).

\begin{tabular}{|c|c|c|c|c|c|c|c|}
\hline \multirow[b]{2}{*}{ Grams } & & \multicolumn{3}{|c|}{ Milliliters } & \multirow{2}{*}{$15 \overline{5}$} & \\
\hline & 58 & 90 & 171 & 332 & 635 & & 2325 \\
\hline 6400 & & & - & & & 514.2 & 476.5 \\
\hline 3200 & & -- & & -- & 212.8 & 235.7 & 228.0 \\
\hline 1600 & & $-\cdots$ & & 109.1 & 88.0 & 91.9 & 80.3 \\
\hline 800 & & 564 & 47.7 & 44.7 & 429 & 39.8 & 31.3 \\
\hline 400 & 27.5 & 29.1 & 25.3 & 22.2 & 20.5 & 17.1 & 13.6 \\
\hline 200 & 14.4 & 13.4 & 14.4 & 11.0 & 9.3 & 7.2 & 5.6 \\
\hline 100 & 7.9 & 6.4 & 5.7 & 4.3 & 3.8 & -- & -- \\
\hline 50 & 3.3 & 3.4 & 2.4 & 2.0 & & & - \\
\hline 25 & 1.7 & 1.3 & -- & & - & & -- \\
\hline
\end{tabular}




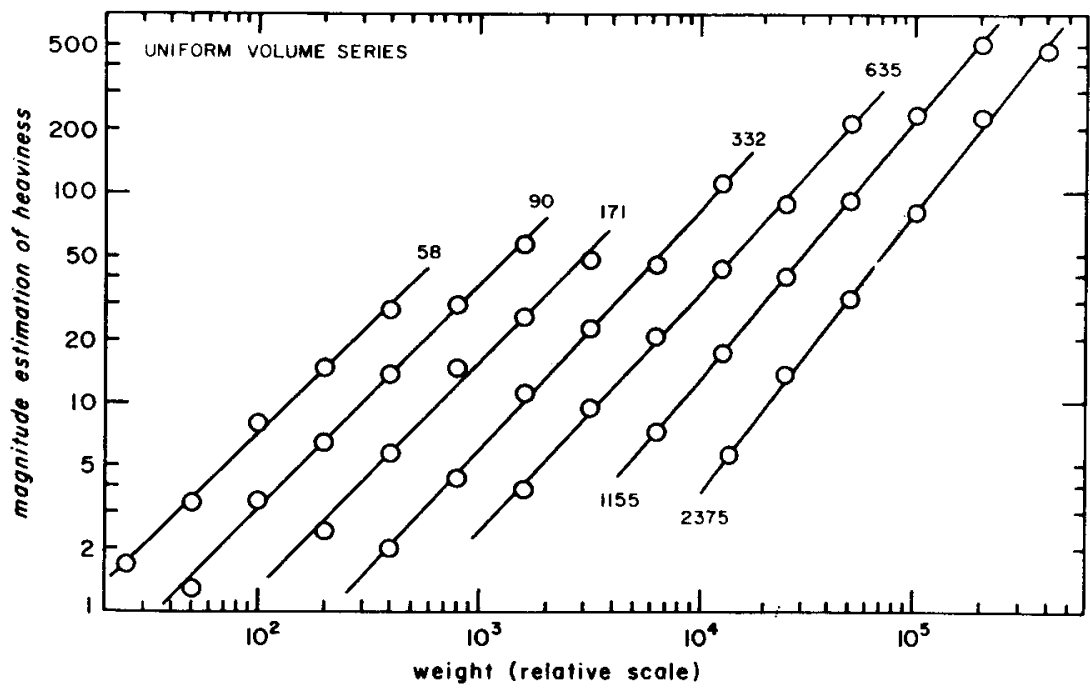

heaviness. In other words, you will try to 'match' numbers to your sensations of heaviness. You may use decimals, fractions, whole numbers, very large numbers. There is no upper or lower limit to the range of numbers available.

"The first few trials will be for practice. You will be told when the experiment actually starts.

"The weights to be estimated differ from one another not only in heaviness but also in volume. Please try to estimate only the heaviness experienced and to disregard the size (volume) of the weight."

The $\mathrm{S}$ was given six practice trials without "feedback." His estimates in these trials were not counted.

\section{RESULTS}

The estimations of heaviness were averaged geometrically across Ss. The geometric means appear in Table 1 and are also plotted in several figures that display various features of the outcome.

Apparent Heaviness as a Function of Weight, Volume Constant

In the log-log coordinates of Fig. 1 the geometric means are plotted as a function of weight. There are seven functions, one for each of the volumes used. For clarity, the positions of the functions were arbitrarily adjusted without altering their form or slope. Each of the seven functions can be fitted well by a straight line, i.e., by a power function whose equation is

$$
\log \psi=\beta \log \varphi+\log \mathrm{k}
$$

Fig. 2. Showing how the constants $\beta$ and $k$ of the power functions for heaviness in Fig. 1 depend on volume. The curves were fitted by a procedure described in the text. The points are from. least-squares solutions using the data in Table 1 . be written:

$$
\psi=\mathrm{k} \varphi^{\beta}
$$
volumes.
Fig. 1. Heaviness as a function of weight. The parameter is volume in milliliters. The abscissa is a relative scale only (the actual stimulus values in grams are stated in Table 1). For clarity the horizontal positions of the ceven functions were arbitrarily adjusted (see text).

weight is measured in grams.

When the seven functions are plotted in their normal (unadjusted) positions and are extrapolated, they tend to converge toward a common point that corresponds to a weight of $8,000-9,000 \mathrm{~g}$. This weight probably approximates the largest that the average $S$ can lift while seated. In fact, many Ss found it very difficult to heft the heaviest stimulus objects $(6,400 \mathrm{~g}=14.1 \mathrm{lb})$.

Given that the functions intersect at the where $\psi$ is apparent heaviness, $\varphi$ is weight, $\beta$ is the slope (exponent), and $k$ is a proportionality constant. Eq. 1 may also

Figure 1 demonstrates that apparent heaviness grows as a power of the physical weight, given any constant volume. This result is not surprising; $S$. S. Stevens and Galanter (1957) earlier showed that power functions are able to describe the results of several independent investigations of heaviness. The various investigators used weights of various (usually constant)

Least-squares solutions reveal that the constants $\beta$ and $\mathrm{k}$ in Fig. 1 vary from one function to another (see Fig. 2). The exponent, $\beta$ increases by about $30 \%$ from smallest to largest volume; $k$ decreases 12 -fold over the same range, given that






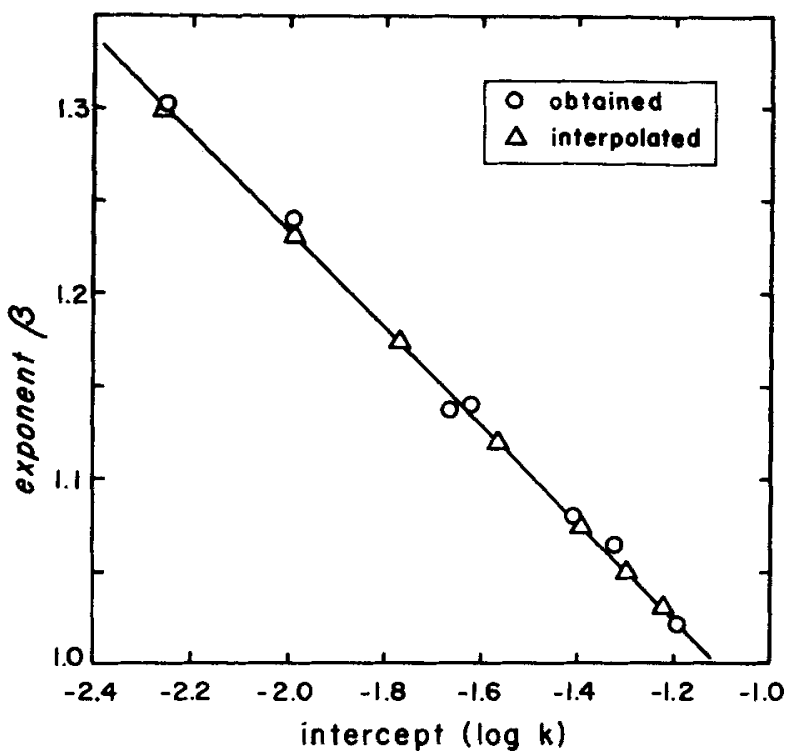

Fig. 3. Showing the relation between $\beta$ and $k$ for the power functions for heaviness. Circles: Values of the constants actually obtained by least-squares solutions using the data in Table 1. Triangles: Values of the constants read from the smooth curves in Fig. 2 (see text). These values were used to construct the theoretical family of heaviness functions shown in Fig. 4.

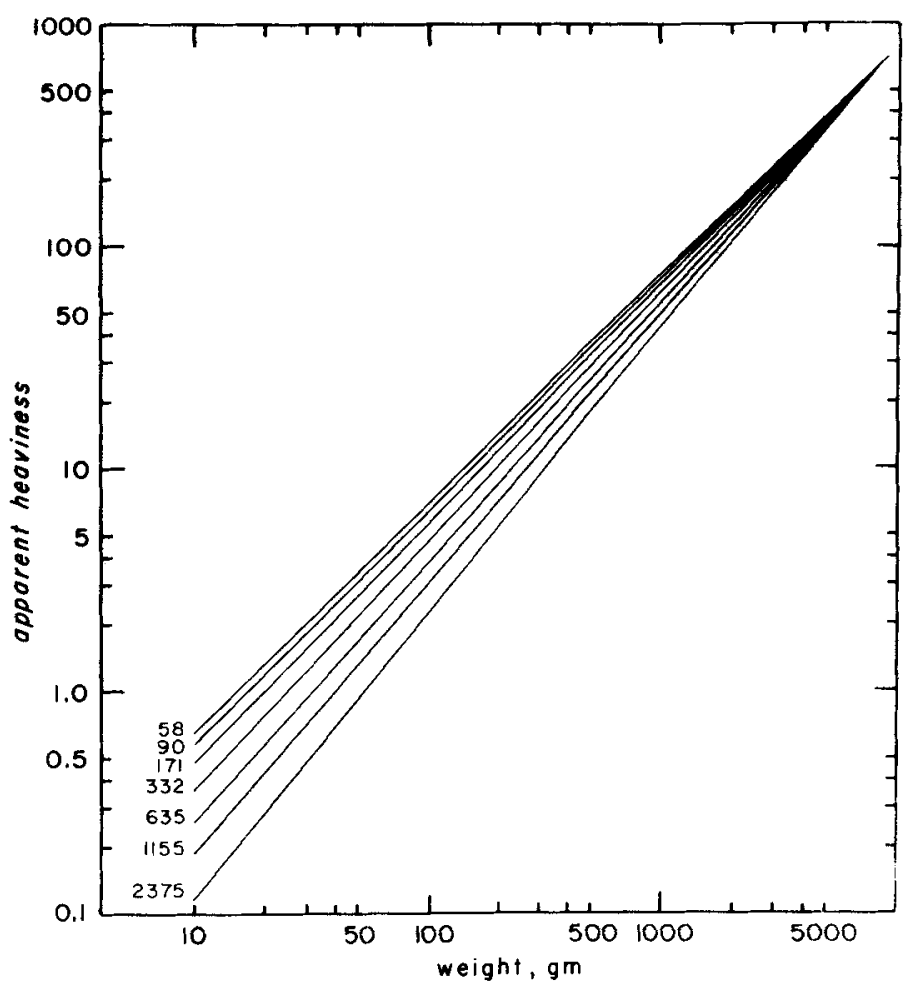

Fig. 4. The idealized family of power functions for heaviness. The parameter is volume in milliliters. constants $(k)$ or, in other words, that $\beta$ is a logarithmic function of $\mathrm{k}$.

To determine whether $\mathrm{Eq} 3$ can describe the present data, the exponents of the seven power functions were plotted in Fig. 3 against the logarithm of $k$. The relatively small deviation of the circles from a straight line argues the likelihood that theoretically the functions do pass through a common point.

The Theoretical Family of Heaviness Functions

functions for constant volume converge suggests that the family of heaviness functions ought to be fitted as a whole, under the assumption that they all share a common point and therefore obey Eq. 3 . In Fig. 2, curves were drawn that both depict the trend of the relation of $\beta$ and $k$ to volume and conform to Eq. 3 , i.e., that make $\beta$ a logarithmic function of $k$. Then, for each volume used in the experiment, new values for $\beta$ and $k$ were read from the smooth curves of Fig. 2. The rationale for this procedure is that, given experimental
The foregoing argument that the

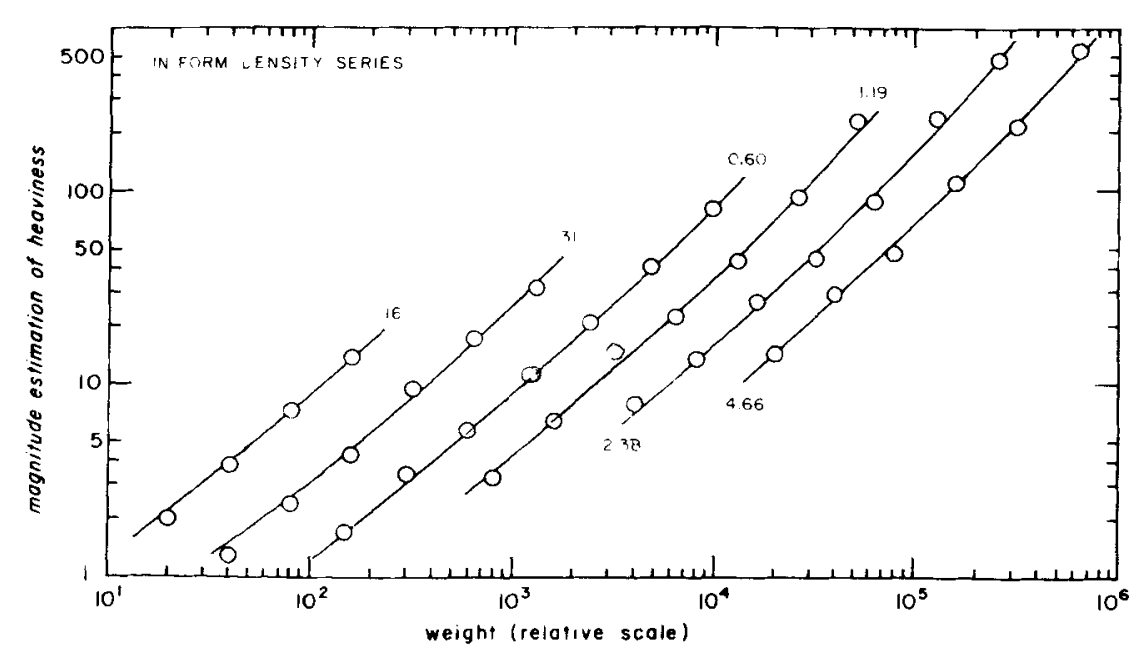
error, the obtained sizes of $\beta$ and $k$ are

Functions subject to random fluctuation, so that it is better to estimate their sizes from the curves in Fig. 2 than to rely solely on the face values obtained by individual least-squares solutions. (By definition the inferred sizes of $\beta$ and $k$ must obey Eq. 3; the triangles in Fig. 3 show this.) Finally, Fig. 4 displays the theoretical family of seven functions drawn with the revised constants.

That the size of the exponent varies with volume may help to explain part of the variation in the results from some eight studies of heaviness reviewed by $S$. S. Stevens and Galanter (1957). But other factors play a part, perhaps especially the variety of scaling methods employed. The eight functions ranged in slope (exponent) from 1.13 to 2.07 . Also, the average, about 1.45 , is larger than the average of the present study. Of the eight studies, the one that agrees best with the present study used the same scaling method, magnitude estimation without a designated standard. Because of the ubiquitous "regression bias" (S. S. Stevens \& Greenbaum, 1966), the method of magnitude estimation can be

Fig. 5. Heaviness as a function of weight. The parameter is density in grams/milliliter. The designated density is the average for the stimulus series since density varied somewhat from stimulus to stimulus. 


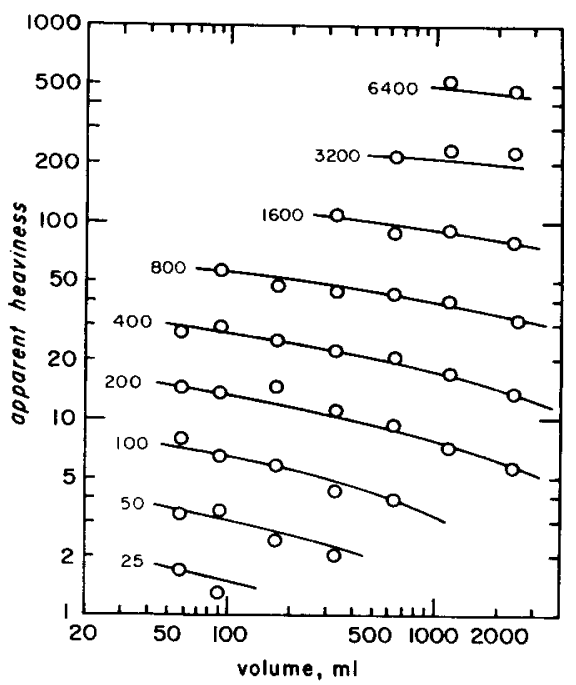

Fig. 6. Heaviness as a function of volume, depicting the size-weight illusion in log-log coordinates. The parameter is weight in grams.

counted on to yield a relatively low exponent. Magnitude production would yield a higher exponent. Despite the regression effect, however, a given scaling method can serve to isolate and quantify one substantial source of variation, namely choice of volume. The precise absolute size of the exponents is another matter.

Figure 4 shows that the smaller the volume, the smaller the exponent of the power function. This suggests the possibility of a limiting theoretical function for which volume is imperceptible. If all cues to volume were eliminated (a condition not so easily arranged as one might think), it is possible that the exponent of the heaviness function would turn out to be smaller than any of those in Fig. 4.

\section{Apparent Heaviness as a Function of} Weight, Density Constant

Any diagonal in Table 1 specifies a set of stimulus objects that have roughly the same density (i.e., volume is proportional to weight). In fact, density varies slightly along a diagonal, but the variation is small compared with the large variation in any column. How does heaviness grow with weight when density is kept constant?

In Fig. 5, the geometric means in Table 1 are plotted again, this time for the several diagonal series. For clarity, the functions have been arbitrarily displaced from one another. In log-log coordinates, they are systematically concave upward in shape. Under conditions of uniform density, apparent weight does not, therefore, seem to grow as a simple power function of weight. This finding is also implicit in the functions for uniform volume depicted in Fig. 4. The curves in Fig. 5 were, in fact, derived from Fig. 4, and they appear to describe adequately the trend of. the points in Fig. 5. It can be demonstrated that if the functions for uniform volume are converging power functions, a uniform density series cannot strictly obey the power law. On the other hand, a hypothetical series from which all cues to apparent volume are absent, thereby eliminating density as a consideration, could theoretically obey the power law.

Apparent Heaviness as a Function of Volume, Weight Constant

The concern here is to describe the size-weight illusion quantitatively over the dynamic range of heaviness. In other words, for any constant weight, how does apparent heaviness change with increasing volume? In Fig. 6, the geometric means from Table 1 are plotted against volume in $\log -\log$ coordinates. They determine a family of functions, spanning a large range of weights, that feature the nature of the size-weight illusion in its practical totality.

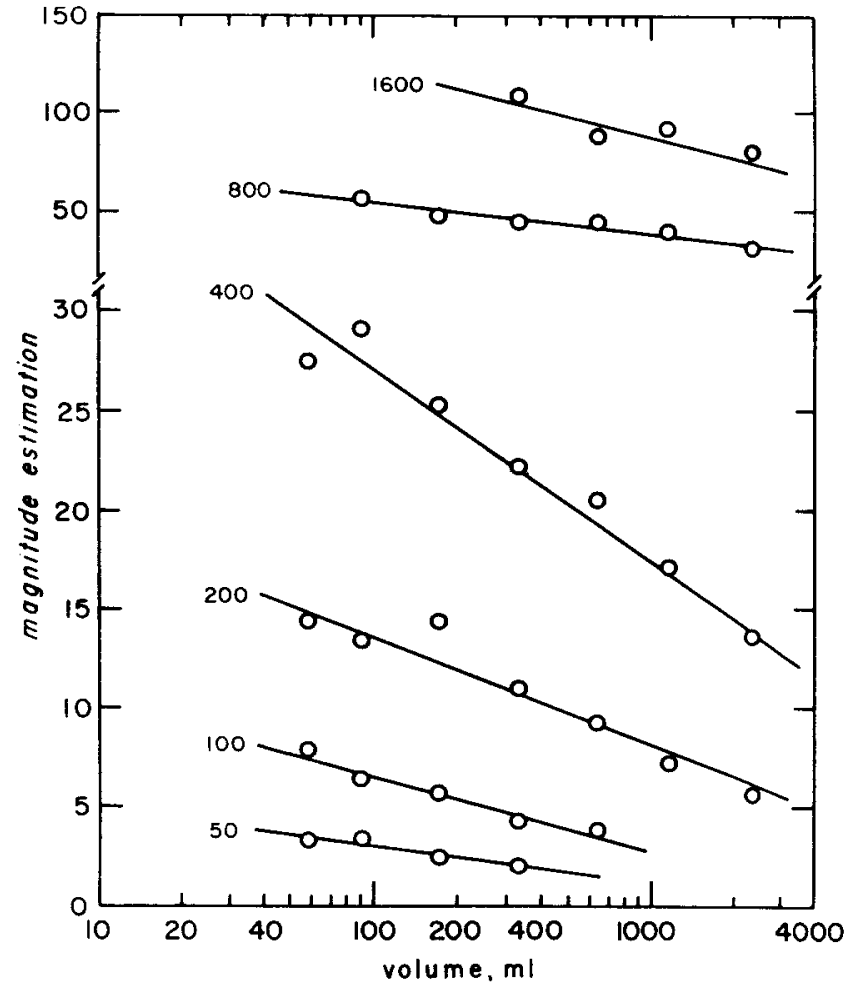

Fig. 7. Heaviness as a function of volume, depicting the size weight illusion in semilog coordinates. The parameter is weight in grams.

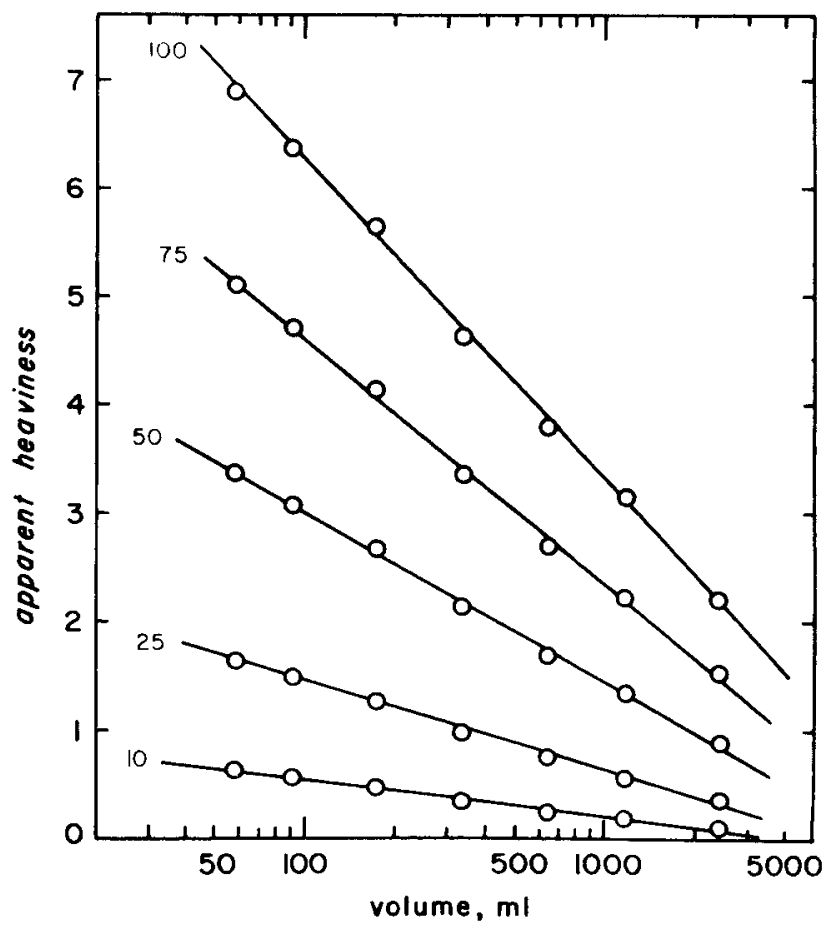

Fig. 8. Heaviness as a function of volume. The points are the intersections of five vertical lines with the family of power functions for heaviness in Fig. 4. The parameter is weight in grams. 


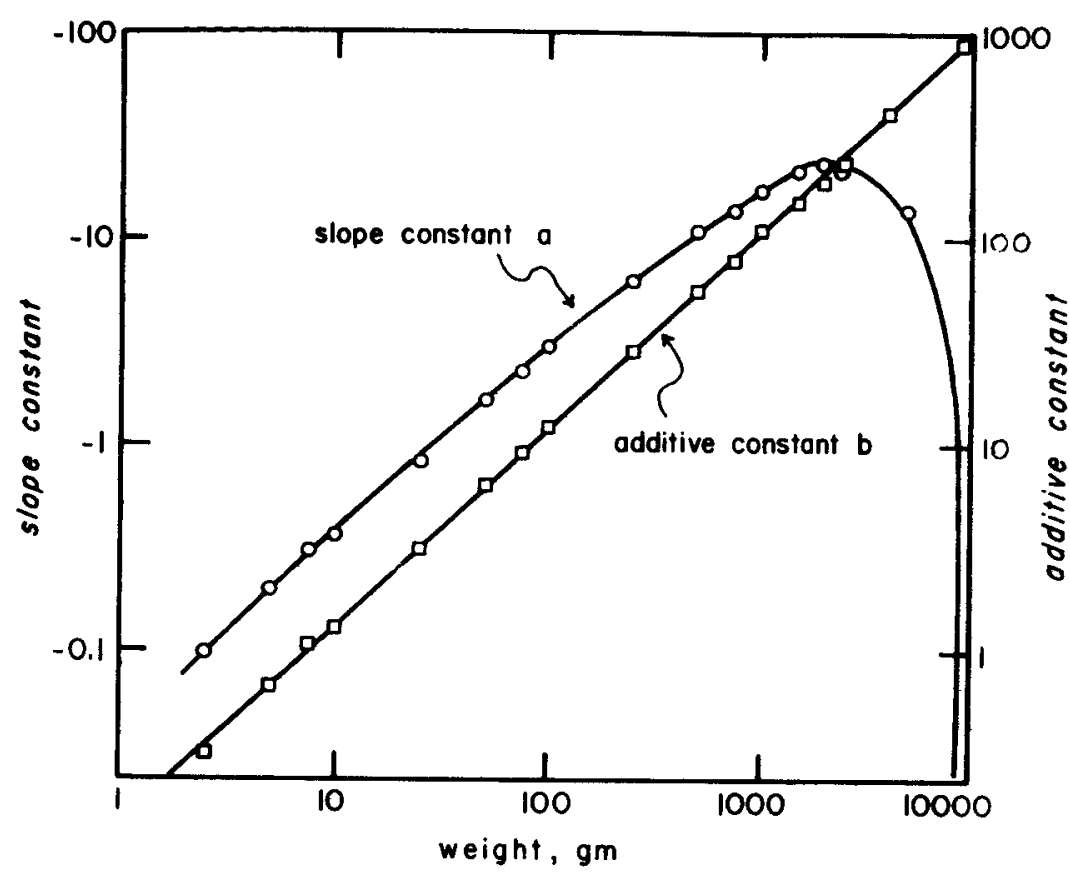

The curves in Fig. 6 were derived from the theoretical family of heaviness functions in Fig. 4 and appear to fit the data adequately. The data cannot be fitted well by a series of power functions or linear functions. On the other hand, a series of logarithmic functions is able to describe the data well and to conform with the theoretical functions in Fig. 4.

Figure 7 presents apparent heaviness as a function of log volume for several different representative weights. Unfortunately, the slopes of the straight lines fitted to the data change so rapidly with weight that it is impossible to portray clearly all of the obtained data in the same graph. (Despite the superficial impression, the slopes in Fig. 7 increase from the smallest to the largest weights. The apparent reversal at $800 \mathrm{~g}$ is simply due to a change of unit on the ordinate.)

Of greater interest than the data points themselves may be the inferred nature of the size-weight illusion as revealed by vertical cuts of the theoretical family in Fig. 4. A vertical cut predicts the loss of heaviness as a constant weight increases in volume. Figure 8 presents a sample of five vertical cuts plotted in semilog coordinates to demonstrate the approximately logarithmic nature of the size-weight illusion. The same picture is obtained when cuts are made anywhere within the span of weight used in the experiment and also in extrapolated portions well above and

Fig. 10. Two theoretical heaviness functions (volume constant) in arithmetic coordinates. risms of equal weight ( $500 \mathrm{~g})$ over a range from 200 to $800 \mathrm{ml}$. Although they
Fig. 9. Showing how the constants of the logarithmic function depicting the size-weight illusion depend on weight.

described the illusion as a power function, a plot of their data in semilog coordinates showed that a $\log$ function probably does as well or better. The range is too short to tell for sure. One lesson learned from the present study is that second-order influences on the perception of heaviness are best revealed by examining them over as large a range as possible.

The family of power functions in Fig. 4 and the family of logarithmic functions in Fig. 8 bring to mind a formally similar finding in visual psychophysics. S. S. Stevens and Diamond (1965) found that the exponent of the power function that relates the apparent brightness of a target to its luminance increases in size when a glare source is introduced into the visual field. The closer the glare is to the target, the greater is the increase in the exponent. Like the family in Fig. 4, the power functions for brightness converge at a common point (in this case at a point that corresponds to the luminance of the glare). The brightness of a target of constant luminance increases as a logarithmic function of the glare angle (distance between the target and the glare source).

Although the family of logarithmic functions serves well to describe the size-weight illusion, the log function should probably be regarded as a highly workable approximation rather than as an exact mathematical specification. Strictly speaking, if the heaviness functions are converging power functions, it can be demonstrated mathematically that the illusion functions cannot be perfectly

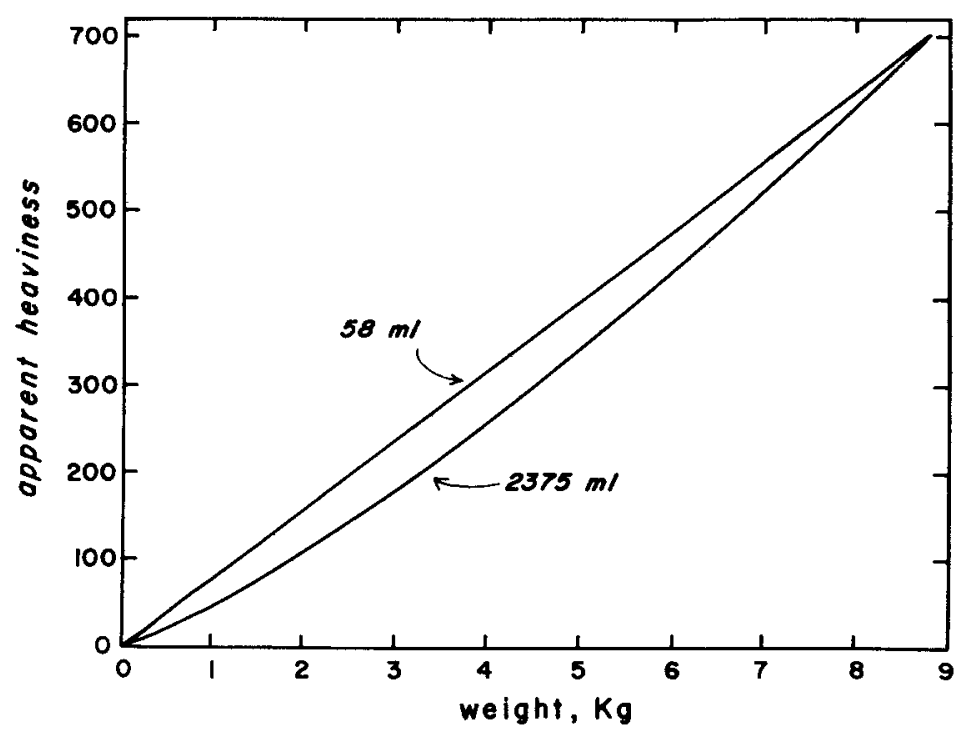


logarithmic. The departures from $\log$ functions are, however, too small to detect graphically within the ranges explored in this experiment.

Figure 9 reveals something about the nature and magnitude of the size-weight illusion. The slope constant a rises steadily with weight up to about $2,000 \mathrm{~g}$. Thereafter it falls. The additive constant $b$ (intercept) grows steadily and almost in direct proportion to weight. The combined effect of the two constants is that the unit loss of apparent heaviness with increased volume is minimal at the upper and lower limits of our experience with weight and maximal for medium weights. The same point is made by Fig. 10, which portrays in arithmetic coordinates two theoretical heaviness functions (for the smallest and largest volumes in the experiment) over the entire range from 0 to about $8,800 \mathrm{~g}$. On the other hand, the percentage loss of heaviness with volume appears to diminish steadily from low to high weights. This is borne out by the progressive flattening of the size-weight functions plotted in the log-log coordinates of Fig. 7 and by the ever-diminishing vertical separation of the power functions in Fig. 4 with increased weight.

\section{REFERENCES}

FRIES, I., \& HOLMBERG, L. The psychophysics of the size-weight illusion. I. Functional relation between heaviness and volume estimations. Psychological Research Bulletin (Lund University, Sweden), 1967, 7, 1-20.

KOSELEFF, P. Studies in the perception of heaviness. I. Some relevant facts concerning the size-weight-effect (SWE). Acta Psychologica, 1957-8, 13, 242-252.

STEVENS, J. C., \& CAIN, W. S. Effort in isometric muscular contractions related to force level and duration. Perception \& Psychophysics, in press.

STEVENS, J. C., \& HALL, J. W. Brightness and loudness as functions of stimulus duration. Perception \& Psychophysics, 1966, 1 , 319-327.

STEVENS, J. C., \& MARKS, L. E. Subjective warmth in relation to the density, duration, and areal extent of infra-red irradiation. Proceedings of the American Society of Heating, Refrigeration, and Air-Conditioning Engineers, in press.

STEVENS, S. S., \& DIAMOND, A. L. Effect of glare angle on the brightness function for a small target. Vision Research, 1965, 5, 649-659.
STEVENS, S. S., \& GALANTER, E. H. Ratio scales and category scales for a dozen perceptual continua. Journal of Experimental Psychology, 1957, 54, 377-411.

STEVENS, S. S., \& GREENBAUM, H. B. Regression effects in psychophysical judgment Perception \& Psychophysics, 1966, 1 439-446.

WARREN, R. M., \& WARREN, R. P. Effect of the relative volume of standard and comparison-object on half-heaviness judgments. American Journal of Psychology, $1956,69,640-643$.

WOLFE, H. K. Some effects of size on judgments of weight. Psychological Review, 1898, 5, 25-54.

\section{NOTES}

1. This research was supported by Contract No. F44620-67-C-0017 with the U.S. Air Force Office of Scientific Research. This research was conducted while the second author held a fellowship in the National Science Foundation Undergraduate Research Participation Program.

2. Address: John B. Pierce Foundation Laboratory, New Haven, Connecticut 06519.

3. Present address: Department of Psychology, Cornell University, Ithaca, New York 14850.

(Accepted for publication December 30, 1969.) 